\title{
Management of Shepherd's Crook Deformity by Proximal Femoral Osteotomy without Bone Graft - A Case Report
}

\section{Avijit Basak, Kunal Mondal*, Pinaki Das, Raunak Agarwal and Subhasish Manna}

Department of Orthopaedics, Institute of Post Graduate Medical Education and Research and Seth Sukhlal Karni Memorial Hospital, West Bengal, India

*Corresponding Author: Kunal Mondal, Department of Orthopaedics, Institute of Post Graduate Medical Education and Research and Seth Sukhlal Karni Memorial Hospital, West Bengal, India.
Received: November 01,2021

Published: December 14, 2021

(C) All rights are reserved by Kunal Mondal., et al.

\section{Abstract}

Introduction: Fibrous dysplasia is a benign condition of the skeletal system. In this disorder there is fibro-osseous proliferation with intervening areas of normal or immature bone in the intramedullary region. This is a case report reflecting management a case of unilateral shepherd's crook deformity at right proximal femur with microfractures causing functional restrictions.

Case Report: A 19-year-old female presented with pain and shortening of right lower limb. Radiographs and histopathology suggestive of fibrous dysplasia. On the right side, we did a valgus close wedged osteotomy in the subtrochanteric region and fixed with dynamic condylar screw supported by 95-degree barrel plate without introducing any bone graft. Patient was followed up for 1 year and ends up with a better functional outcome evaluated by Harris hip score and Oxford hip score.

Conclusion: Lateral close wedged osteotomy is effective method to correct the shepherd's crook deformity if properly executed. Hence using an extramedullary device without bone graft can be the choice of treatment in such cases.

Keywords: Fibrous Dysplasia; Shepherd's Crook Deformity; Lateral Close Wedged Osteotomy; Dynamic Condylar Screw; 95-Degree Barrel Plate; Extramedullary Device; Bone Graft

\section{Introduction}

Fibrous dysplasia is an intrinsic defect of enchondral bone maturation that results in an incomplete or immature ossification pattern. This abnormal pattern is characterized by a benign fibrous stroma stippled with bony islands of metaplastic bone, resulting in structural weakening of the bone and pathological fractures [1]. Deformities lead to both cosmetic and functional restrictions. The most common sites of skeletal involvement in fibrous dysplasia are the ribs, proximal femur, craniofacial bones, posterior maxilla [2]. The pathology may involve only a small segment of bone, or it may occupy its entire length. Incidentally discovered fibrous dysplasia which are asymptomatic causing no functional and cosmetic hazards, require only clinical observation. Follow-up radiographs with clinical evaluation in every 3 months to look for progression has been recommended. In newly identified cases, radiographs of certain areas like pelvic, spine, periarticular regions along with endocrinal evaluation is required to exclude a diagnosis of polyostotic disease. Bisphosphonates, pamidronate, have been used to decrease bone pain in symptomatic patients with polyostotic disease $[5,6]$. Simple true cut biopsy (multiple samples must) is sufficient to confirm the diagnosis of fibrous dysplasia when there is a nonclassical presentation. Surgical procedures are essential to correct deformities, prevention of pathological fractures, or eradication of symptomatic lesion $[7,8]$. In this study, surgical option was opted for correction of deformity and limb length discrepancy in a known case of fibrous dysplasia of right hip joint. 


\section{Case Report}

A 19-year-old female patient reported to us with progressive deformity over right hip and limb length shortening with pain over right groin. The range of motion is restricted. Surprisingly patient was only symptomatic over right hip region, rather left side became noncompliant. Her right trochanter protruded laterally, and her leg was rotated externally and shortened by 5 -cm (Figure 1A). Radiographs showed a severe varus deformity of the right proximal femur with a large expansile lytic lesion with a patchy area of ground glass haze in the upper meta diaphyseal and femoral neck (Figure $1 B, C$ ), with evidence of a cortical breach suggestive of pathological fracturing. A $50^{\circ}$ varus deformity also present in the femoral neck.

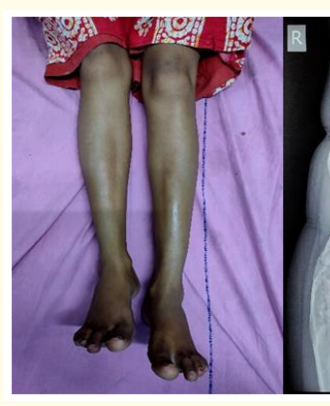

(A)

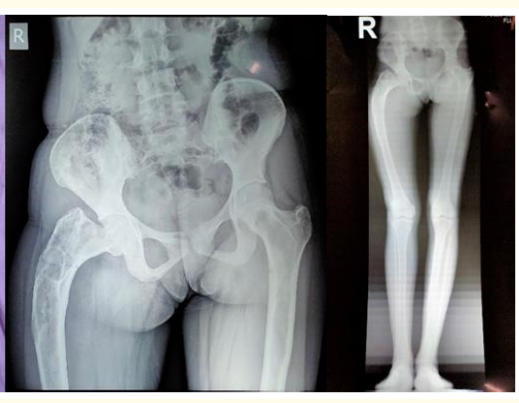

(B)
(C)
Figure 1: A: Limb length discrepancy, B, C: Shepherd crook deformity over right hip region.

\section{Operative procedure and follow ups}

Radiographs were used to determine the appropriate size and location for a closed wedge osteotomy of the proximal femur. The patient was placed in a supine position under an image intensifier and underwent a duo planar osteotomy (Figure 2B) (valgus osteotomy with a $45^{\circ}$ lateral closing wedge) of the subtrochanteric region supported by extramedullary device with angled screw support (Figure 2C). Bone Graft was not used in this case. Initial post operative period was uneventful. Assisted weight bearing was given after 8 weeks with dynamic quadriceps and hamstring exercises. Limb length discrepancy improved post operatively. The final limb length difference came down to $1 \mathrm{~cm}$ (Figure 3A). Range of motion improved in all planes (Figure 3B,C). Radiographic analysis of immediate post operative (Figure 4A) and 12 months postoperative
(Figure 4B) follow up was done with serial intervals. The new neck shaft angle became 138 degrees of right side compared to left one which was 132 degrees. Functional evaluation was done by Harris hip score and Oxford hip scoring systems (Table 1). In Harris hip score the status changed from 31.75 to 88.82 over 1 year follow up. Preoperative oxford hip score was 9 which jumped to 41 after 1 year of rehabilitation (Table 1).

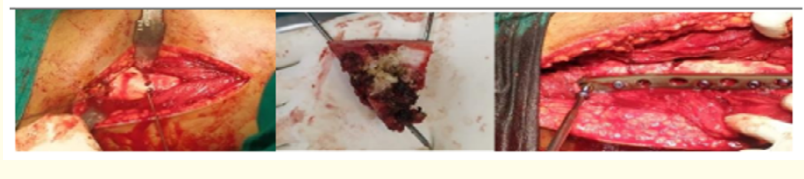

(A)

(B)

(C)

Figure 2: A: Osteotomy site selected, B: wedged portion of bone is cut out, C: fixed with 95- degree angled barrel plate with dynamic condylar screw.

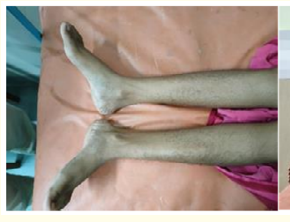

(A)

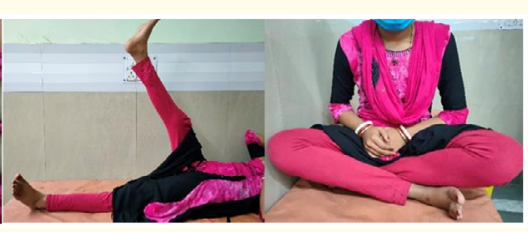

(B)
(C)
Figure 3: A: Length mismatch reduced, B: Straight leg rising test is positive, C: Can be able to do cross leg sitting.

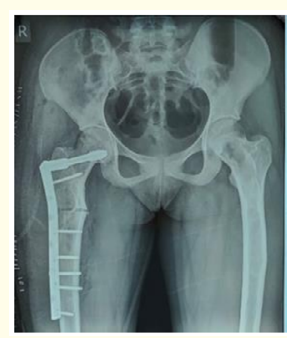

(A)

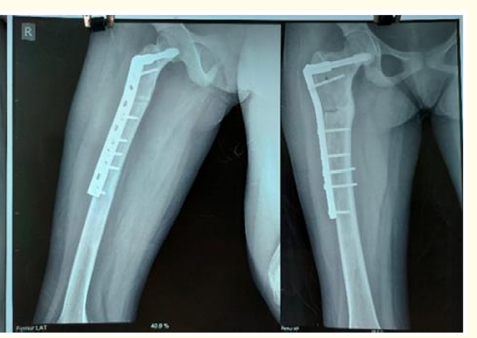

(B)
(C)
Figure 4: A: Immediate postoperative, B: Scannogram of 1 year follow up. 


\begin{tabular}{|l|c|c|}
\hline Status & Preoperative & Postoperative [1 Year] \\
\hline Harris hip score & 31.75 percentile & 88.82 percentile \\
\hline Oxford hip score & 9 & 41 \\
\hline
\end{tabular}

Table 1: Functional evaluation both pre and postoperative phase.

\section{Discussion}

Fibrous dysplasia is a disorder of bone where normal medullary cavities of multiple bones are replaced by fibro-osseous tissue containing trabeculae of newly formed primitive bone [9]. Pathology of Fibrous dysplasia can affect the bones at all stages of growth process. Fibrous dysplasia lesions are more commonly found in proximal femur and tends to produce bowing and varus deformity due to constant muscle pull and body weight on the weakened bone [10]. The shepherd's crook deformity is characteristics feature of fibrous dysplasia presents with pain, limb length discrepancy, limp, and impending femoral neck fractures. Other common sites include the tibia, skull and ribs, although any bone can be affected [11]. The mainstay of surgical treatment is to restore normal alignment of the bone, restore normal walking ability and to provide pain relief. Several procedures have been advocated for treating proximal femoral lesions in fibrous dysplasia, including curettage and bone grafting, valgus osteotomy, plating and hip nailing, intramedullary nailing, and cortical bone grafting [12]. However, the type of intervention depends on many factors such as patient age, lesion characteristics (site, size and biological behavior) and the presence of deformity [13]. Conservative modality such as second and third generation bisphosphonates plays a crucial role in maintaining the strength of the bone, pain relief and lowers the incidence of stress fractures which has been reserved for selected patients $[14,15]$ (Figure 4). The main cause for shepherd's crook deformity is the mechanical stress during weight bearing through the weak bone. So, it is essential to provide some mechanical support in form of internal/external fixation, although disease progression cannot be altered [16]. Breck [17] reported a case of fibrous dysplasia treated with total femoral plating and hip nailing, without further fracture or subsequent implant failure. Connolly [18] and Freeman., et al. Reported the use of osteotomies with Zickel nail fixation [19]. There are various types of internal fixation for treating shepherd's crook deformity but none of them are superior to other. Moreover, deformity often spared the femoral head. So, firm purchase of implant in the femoral head provides sufficient mechanical support and prevent recurrence of the deformity [20]. The side plate barrel should be long enough to provide adequate fixation of the mechanically deficient femur and to prevent implant failure. In our cases, fixation was achieved with a 10-hole side plate. In our experience, dynamic condylar screw fixation with angled plate barrel provides a more versatile method of fixing the shepherd's crook deformity of proximal femur. The $95^{\circ}$ plate with barrel will act as a template. As various studies have showed that fibrous dysplasia possesses a normal bone healing bone grafting is not always necessary for bony union. Therefore, we did not make any efforts to do an additional bone grafting in our present study [22]. A gamma nail may be another option, because it can also provide good mechanical support over the femoral neck. In addition, it possesses a shorter level arm than a dynamic condylar screw and has a lower bending moment on the femoral neck. Moreover, a gamma nail is difficult to introduce, and may easily protrude from the canal because of the difficulty in locating a good entrance point and the deformed proximal femur [21]. It is difficult to achieve single stage correction using a long intramedullary implant as the femur is excessively bowed, and in such cases, dynamic condylar screw fixation should be preferred. Thus, lateral close wedged osteotomy with dynamic condylar screw fixation with 95-degree angled plate barrel is a versatile, effective, easier, and a practical approach to correct the morbid shepherd's crook deformity of proximal femur avoiding additional bone grafting steps.

\section{Conclusion}

Impairments caused by shepherd's crook deformity can be overcome both functionally and cosmetically by valgus osteotomy with a well-supported extramedullary device. Use of bone graft is not necessary always.

\section{Clinical Message}

Extramedullary device without additional bone graft can be an easy alternative to achieve alignment in shepherd crook deformities in hip.

\section{Bibliography}

1. Alman BA., et al. "Activating mutations of Gs protein in monostotic fibrous lesions of bone". Journal of Orthopaedic Research 14.2 (1996): 311-315.

2. DiCaprio MR and Enneking WF. "Fibrous dysplasia. Pathophysiology, evaluation, and treatment". Journal of Bone and Joint Surgery 87.8 (2005): 1848-1864. 
3. Wei-Jen Chen, Wei-Ming Chen, Chao-Ching Chiang, Ching-Kuei Huang, Tain-Hsiung Chen, Wai-Hee Lo. Journal of the Chinese Medical Association 68.7 (2005): 343-346.

4. Albright F., et al. "Syndrome characterized by osteitis fibrosa disseminata, areas of pigmentation and endocrine dysfunction, with precocious puberty in females". New England Journal of Medicine 216 (1937): 727-746.

5. Chapurlat RD., et al. "Treatment of fibrous dysplasia of bone with intravenous pamidronate: long-term effectiveness and evaluation of predictors of response to treatment". Bone 35.1 (2004): 235-242.

6. Liens D., et al. "Long-term effects of intravenous pamidronate in fibrous dysplasia of bone”. Lancet 343. 8903 (1994): 953954.

7. Bernando Vagars. “Orthopaedic surgery for Fibrous Dysplasia medicine". Medscape (2016).

8. Guille JT., et al. "Fibrous dysplasia of the proximal part of the femur. Long-term results of curettage and bone-grafting and mechanical realignment". Journal of Bone and Joint Surgery 80.5 (1998): 648-658.

9. Lee SE., et al. "The diagnostic utility of the GNAS mutation in patients with fibrous dysplasia: meta-analysis of 168 sporadic cases". Human Pathology 43.8 (2012): 1234-1242.

10. DiCaprio MR and Enneking WF. "Fibrous dysplasia. Pathophysiology, evaluation, and treatment". Journal of Bone and Joint Surgery 87.8 (2005): 1848-1864.

11. Russell LW and Chandler FA. "Fibrous dysplasia of bone". Journal of Bone and Joint Surgery 32.2 (1950): 323-337.

12. Enneking WF and Grearen PF. "Fibrous dysplasia of the femoral neck. Treatment by cortical bone-grafting". Journal of Bone and Joint Surgery 68.9 (1986): 1415-1422.

13. Lane JM., et al. "Bisphosphonate therapy in fibrous dysplasia". Clinical Orthopaedics and Related Research 382 (2001): 6-12.

14. Chapurlat RD., et al. "Long-term effects of intravenous pamidronate in fibrous dysplasia of bone". Journal of Bone and Mineral Research 12.10 (1997): 1746-1752.

15. Ozaki T., et al. "Polyostotic fibrous dysplasia: a long term follows up of 8 patients". International Orthopaedics 20.4 (1996): 227-232.
16. Breck LW. "Treatment of fibrous dysplasia of bone by total femoral plating and hip nailing". Clinical Orthopaedics 82 (1972): 82-83.

17. Connolly JF. "Shepherd's crook deformity of polyostotic fibrous dysplasia treated by osteotomy and Zickel nail fixation". Clinical Orthopaedics and Related Research 123 (1977): 22-24.

18. Freeman BH., et al. "Multiple osteotomies with Zickel nail fixation for polyostotic fibrous dysplasia involving the proximal part of the femur". Journal of Bone and Joint Surgery 69.5 (1987): 691-698.

19. Guille JT., et al. "Fibrous dysplasia of the proximal part of the femur: long-term results of curettage and bone-grafting and mechanical realignment". Journal of Bone and Joint Surgery 80.5 (1998): 648-658.

20. Yamamoto T., et al. "Oblique wedge osteotomy for femoral diaphyseal deformity in fibrous dysplasia". Clinical Orthopaedics 384 (2001): 245-249.

21. Enneking WF. "Fibrous lesion originating in bone. In Musculoskeletal Tumor Surgery". New York: Churchill- Livingstone (1983): 855-867.

22. Campanacci M. "Fibrous dysplasia in Bone and Soft Tissue Tumors, $2^{\text {nd }}$ edition". New York: Springer-Verlag Wien (1999): 435-461.

\section{Assets from publication with us}

- Prompt Acknowledgement after receiving the article

- Thorough Double blinded peer review

- Rapid Publication

- Issue of Publication Certificate

- High visibility of your Published work

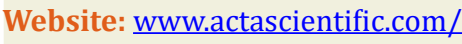

Submit Article: www.actascientific.com/submission.php Email us: editor@actascientific.com

Contact us: +919182824667 